\title{
Evaluating Training Programs for Electroacupuncture Techniques with Skin Temperature as a New Index
}

\author{
Tomoko Kubota1, Hidetoshi Mori'1,2, Tateyuki Morisawa ${ }^{3}$, Kazuyo Hanyu ${ }^{2,4}$, \\ Hiroshi Kuge ${ }^{2,5}$, Mayumi Watanabe ${ }^{2,6}$, Tim Hideaki Tanaka² \\ ${ }^{1}$ Division of Health Sciences, Graduate School of Technology and Science, Tsukuba University of Technology, Tsukuba, Japan \\ ${ }^{2}$ Department of Health, Faculty of Health Sciences, Tsukuba University of Technology, Tsukuba, Japan \\ ${ }^{3}$ Graduate School of Life and Environmental Sciences, University of Tsukuba, Tsukuba, Japan \\ ${ }^{4}$ Faculty of Life and Environmental Sciences, University of Tsukuba, Tsukuba, Japan \\ ${ }^{5}$ Department of Anesthesiology Pain Clinic, Osaka Medical College Hospital, Osaka, Japan \\ ${ }^{6}$ Department of Science and Engineering, Chuo University, Tokyo, Japan \\ Email: watanabem62@gmail.com
}

How to cite this paper: Kubota, T., Mori, H., Morisawa, T., Hanyu, K., Kuge, H., Watanabe, M. and Tanaka, T.H. (2017) Evaluating Training Programs for Electroacupuncture Techniques with Skin Temperature as a New Index. Health, 9, 1589. 1596.

https://doi.org/10.4236/health.2017.911116

Received: July 18, 2017

Accepted: October 27, 2017

Published: October 30, 2017

Copyright ( 2017 by authors and Scientific Research Publishing Inc. This work is licensed under the Creative Commons Attribution International License (CC BY 4.0).

http://creativecommons.org/licenses/by/4.0/

\begin{abstract}
Training in acupuncture techniques has a long history of thousands of years. It has been individually handed down from person (teacher) to person (student). However, techniques and training have not been scientifically evaluated because individual differences may exist among evaluators. In animal studies, some researchers have reported that acupuncture stimulation dilates blood vessels of the skin and skeletal muscles. These studies also reveal an association between skin temperature (ST) and blood circulation volume on the skin. Our previous studies have reported that acupuncture stimulation, especially that of electroacupuncture (EA), can elevate ST. Therefore, we monitored the instructive effects and level of EA techniques with ST and propose that we can bring monitoring ST into training/education of EA as a new index of technical assessment. Moreover, ratio of changes might be used as new criteria for retraining. Healthy students $(\mathrm{n}=14)$ were given with 10 minutes of EA stimulation on the tibialis anterior: Zusanli (ST36) and Tiaokou (ST38). Their ST was monitored before and during stimulation as well as for 30 minutes after stimulation. All subjects showed a nominal increase in ST. At the time, ratios of changes were also calculated. Two subjects did not reach the average of $1.3 \%$. This suggests that the technical level of the therapist was inadequate. Thus, observation of ST elevation and calculation of the average ratio of ST change (elevation) could be applied to a new scientific index of technical assessment in acupuncture treatment training. However, further research (e.g., larger-scale studies, adjustment for gender differences, or other age subjects)
\end{abstract}


is required to support these findings.

\section{Keywords}

Electric Acupuncture (EA), Skin Temperature (ST), Training/Education, Evaluation

\section{Introduction}

Acupuncture has a long history and is widely practised worldwide [1]. One of the oldest findings was the Tyrolean Iceman, who had tattoos at acupuncture points, which suggests the possible origins of acupuncture [2].

In other words, this wisdom of the ancients was handed down from generation to generation for thousands of years. Sometimes it was passed on in the Classics, such as the Yellow Emperor's Classics of internal medicine, and sometimes it was individually handed down from person (teacher) to person (student).

Nowadays, the knowledge and techniques are taught in colleges and universities so that students may obtain national certification. Schools provide a systematized curriculum and textbooks. They also investigate the effectiveness of instruction and have developed many evaluation sheets or check lists [3] [4]. However, scientific evaluation of effectiveness is not easy because individual differences may exist among evaluators.

In animal studies, some researchers have reported that acupuncture stimulation dilates blood vessels of the skin and skeletal muscles [5] [6] [7]. These studies also reveal an association between skin temperature (ST) and blood circulation volume on the skin [8] [9].

Previously, we studied whether the effectiveness of electroacupuncture (EA) could be evaluated by changes in ST. We also investigated whether EA elevated ST by various methods such as thermographic laser Doppler velocimetry, near-infrared spectroscopy, and hydrogen clearance and we reported that EA would definitely elevate ST [5] [10] [11] [12] [13].

In this study, we monitored the effectiveness of instruction and level of EA techniques with ST and we propose that we can bring monitoring of ST into training/education of EA as a new index of technical assessment. Moreover, ratio of changes might be used as new criteria for retraining.

\section{Materials and Methods}

\subsection{Subjects}

Healthy students $(\mathrm{n}=14($ male $=12$, female $=2)$, average $23.5 \pm 2.7$ years old $)$ participated in this study. 2nd year students of acupuncture and moxibustion course were recruited. A total of 14 students participated. All of them had sufficient training, knowledge and techniques of EA in the course with experienced 
acupuncturists (Table 1).

Subjects worked in pairs; one subject provided EA stimulation to the other. Next, the roles were reversed. At the same time, they also monitored changes in ST (Figure 1(a)).

This study adhered strictly to the principles of the Declaration of Helsinki. One week before the study began, the participants were informed of the purpose and procedures of the study, and all of them signed the consent form. This study was approved by the institutional review board of the Tsukuba University of Technology, Tsukuba, Japan (H28-01).

\subsection{Methods}

The protocol of this study is illustrated in Figure 1(b).

\subsubsection{EA Stimulation}

Subjects rested in the supine position for 15 minutes before they received EA stimulation $(1 \mathrm{~Hz})$ for 10 minutes using two disposable acupuncture needles (40 $\mathrm{mm}$ in length, $0.20 \mathrm{~mm}$ in diameter, Seirin Corporation, Shizuoka, Japan) at a depth of 10 - $15 \mathrm{~mm}$ into two acupuncture points on the tibialis anterior: Zusanli (ST36) and Tiaokou (ST38). EA was applied on the right leg. An Ohm Pulser LFP-4000A (Zen Iryoki Corporation, Fukuoka, Japan) was used as the device for generating EA stimulation (H28-01).

\subsubsection{ST Monitoring Methods}

Changes in ST were observed with a monitor, TH-200 (Suzuki Iryoki, Co., Ltd.), in the middle of the two points as shown in Figure 1(c).

Table 1. Subjects.

\begin{tabular}{ccc}
\hline Subject & Age & Gender \\
\hline 1 & 19 & male \\
2 & 21 & female \\
3 & 22 & male \\
4 & 19 & male \\
5 & 19 & male \\
6 & 19 & male \\
7 & 19 & male \\
8 & 20 & male \\
9 & 20 & male \\
10 & 19 & female \\
11 & 21 & male \\
12 & 20 & male \\
13 & 55 & male \\
14 & 36 & male \\
& & \\
\hline & &
\end{tabular}


(a) subject on the left gave EA stimulation to the subject on the right

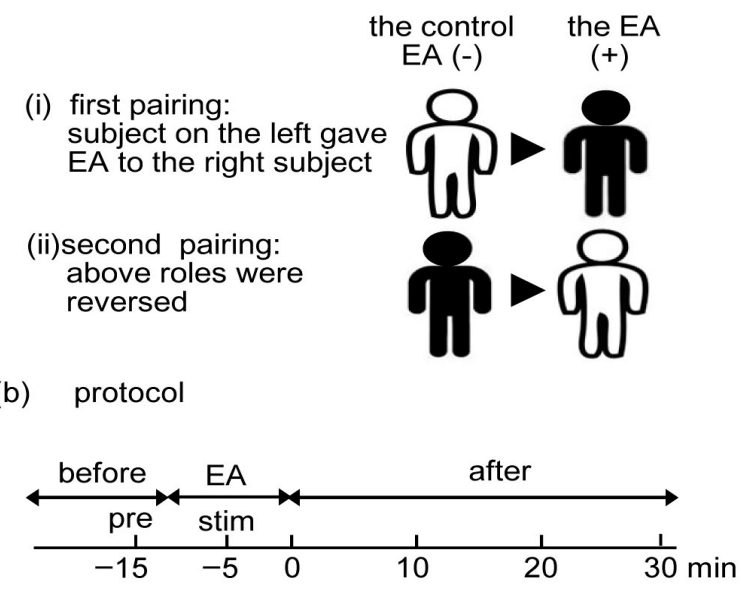

(c) points of

$$
0 \text { : EA stimulation }
$$

$\boldsymbol{\nabla}$ : skin temperature measurement

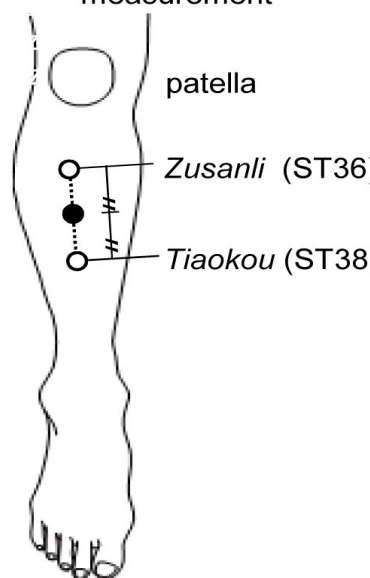

Figure 1. (a-i) First, subjects worked in pairs; one subject (on the left = figure in white) provided EA stimulation to the other (on the right = figure in black). (a-ii) Second, these roles were reserved (the black gave EA stimulation to the white). At the same time, they also monitored changes in ST. (b) In this study, ST was recorded at (pre); 5 minutes after the start of EA (stim); immediately after EA (post 0); and at 5 (post 5), 10 (post 10), 15 (post 15), 20 (post 20), 25 (post 25), and 30 (post 30) minutes after stimulation. (c) EA stimulation and ST measurement points: $\bigcirc$ EA stimulation, $\bigcirc$ skin temperature measurement.

Points of measurements for time were as indicated below: before stimulation (pre), during stimulation (stim), immediately after stimulation (post 0), 5 minutes post-stimulation (post 5), 10 minutes post-stimulation (post 10), 15 minutes post-stimulation (post 15), 20 minutes post-stimulation (post 20), 25 minutes post-stimulation (post 25) and 30 minutes post-stimulation (post 30).

This experiment was conducted in a room at a constant temperature $\left(24.3^{\circ} \mathrm{C}\right.$ $\left.\pm 0.3^{\circ} \mathrm{C}\right)$ and humidity $(31.5 \% \pm 0.8 \%)$.

\subsection{Ratio of ST Changes}

Those ratios were obtained by comparison with ST at the point in time before stimulation (pre) according to the below formula $\Delta \%=$ [pre - (stim, post 0 , post 5 , post 10 , post 15 , post 20 , post 25 , or post 30$) /$ pre] $\times 100$; the average values of $\Delta \%$ were also calculated $(\Delta \%=1.26)$.

\subsection{Statistical Analysis}

Differences between values were determined by two-way analysis of variance followed by post hoc comparisons, Fisher's LSD multiple comparison test with SPSS Advanced Models Version. The level of significance was set at $\mathrm{p}<0.05$ and all data were presented as mean \pm SEM.

\section{Results}

Our results are shown in Figure 2 and Table 2. ST of the EA group showed a 


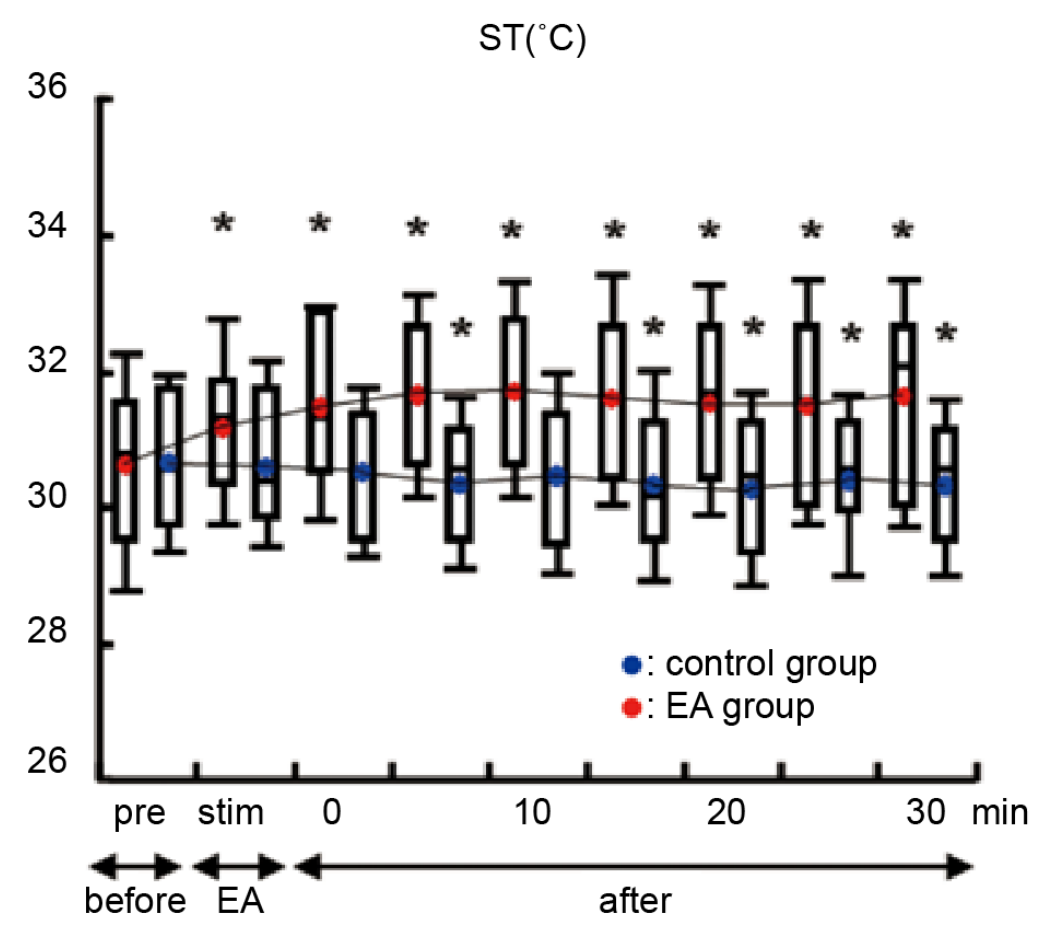

Figure 2. ST of the EA group (O) showed a significant increase, however, the control group (O) did not. ${ }^{*} \mathrm{p}<0.05$ vs pre.

Table 2. Ratio of ST changes (elevations) after EA stimulation.

\begin{tabular}{|c|c|c|c|c|c|c|c|c|c|c|}
\hline Subject \Time & pre & stim & post 0 & post 5 & post 10 & post 15 & post 20 & post 25 & post 30 & average \\
\hline 1 & 0 & 4.2 & 4.2 & 3.2 & 3.2 & 1.8 & 0 & 1.4 & 1.1 & 2.4 \\
\hline 2 & 0 & $\mathbf{\Delta} 2.7$ & $\Delta 1.0$ & 0.7 & 0.7 & 0.3 & 1.7 & 0 & $\Delta 0.3$ & $\Delta 0.1$ \\
\hline 3 & 0 & 3.2 & 3.5 & 2.8 & 3.2 & 2.8 & 2.8 & 2.8 & 2.5 & 3 \\
\hline 4 & 0 & 2.6 & 0.3 & 0.3 & 0.3 & $\Delta 0.3$ & $\mathbf{\Delta} 0.3$ & $\boldsymbol{\Delta} 2.3$ & $\Delta 2.3$ & $\Delta 0.2$ \\
\hline 5 & 0 & 1 & 4.1 & 4.8 & 4.8 & 5.1 & 5.1 & 5.4 & 5.4 & 4.4 \\
\hline 6 & 0 & $\Delta 2.2$ & $\Delta 0.9$ & $\Delta 0.9$ & 0.9 & 0.3 & 0.6 & 0.9 & 1.2 & $\Delta 0$ \\
\hline 7 & 0 & 3.7 & 3.7 & 3.4 & 3.7 & 3.4 & 2 & 1.7 & 2 & 3 \\
\hline 8 & 0 & 3.8 & 5.2 & 6.6 & 6.3 & 5.6 & 4.9 & 5.2 & 4.5 & 5.3 \\
\hline 9 & 0 & 4.5 & 7.3 & 8 & 8.3 & 8.6 & 8.9 & 8.6 & 8.6 & 7.8 \\
\hline 10 & 0 & $\Delta 0.7$ & 2.3 & 3.9 & 4.5 & 2.9 & 2.6 & 1.6 & 3.5 & 2.6 \\
\hline 11 & 0 & 4 & 5.3 & 7 & 6.3 & 5.6 & 6 & 6.6 & 7.6 & 6 \\
\hline 12 & 0 & 2.7 & 4.8 & 6.8 & 6.1 & 6.1 & 6.8 & 7.1 & 8.5 & 6.1 \\
\hline 13 & 0 & 0.6 & $\Delta 1.6$ & $\Delta 0.6$ & $\Delta 1.3$ & $\Delta 1.6$ & $\Delta 1.3$ & $\Delta 0.6$ & 1 & $\Delta 0.7$ \\
\hline 14 & 0 & 0 & 1.6 & 1.2 & 2.8 & 3.1 & 0.9 & 0.9 & 2.5 & 1.6 \\
\hline
\end{tabular}

$(\boldsymbol{\Lambda}$ : negative number).

significant increase from pre $\left(30.6^{\circ} \mathrm{C} \pm 0.3^{\circ} \mathrm{C}\right)$ to stim $\left(31.1^{\circ} \mathrm{C} \pm 0.3^{\circ} \mathrm{C}\right)$, post 0 $\left(31.4^{\circ} \mathrm{C} \pm 0.3^{\circ} \mathrm{C}\right)$, post $5\left(31.6^{\circ} \mathrm{C} \pm 0.3^{\circ} \mathrm{C}\right)$, post $10\left(31.7^{\circ} \mathrm{C} \pm 0.4^{\circ} \mathrm{C}\right)$, post 15 $\left(31.5^{\circ} \mathrm{C} \pm 0.4^{\circ} \mathrm{C}\right)$, post $20\left(31.5^{\circ} \mathrm{C} \pm 0.4^{\circ} \mathrm{C}\right)$, post $25\left(31.4^{\circ} \mathrm{C} \pm 0.4^{\circ} \mathrm{C}\right)$, and post 30 
$\left(31.6^{\circ} \mathrm{C} \pm 0.4^{\circ} \mathrm{C}\right)$ respectively $(\mathrm{p}<0.05)$.

However, the control group did not show such elevation; pre $\left(30.6^{\circ} \mathrm{C} \pm 0.3^{\circ} \mathrm{C}\right)$ to post $5(30.3 \pm 0.3)$, post $15\left(30.3^{\circ} \mathrm{C} \pm 0.4^{\circ} \mathrm{C}\right)$, post $20\left(30.2^{\circ} \mathrm{C} \pm 0.3^{\circ} \mathrm{C}\right)$, post 25 $\left(30.4^{\circ} \mathrm{C} \pm 0.3^{\circ} \mathrm{C}\right)$, and post $30\left(30.3^{\circ} \mathrm{C} \pm 0.4^{\circ} \mathrm{C}\right)$. At five points in time, (post 5 and post 15 - post 30 ), ST was significantly lower than that of pre (before the experiment) (Figure 2).

\section{Discussion}

ST increased at all points in time for all subjects after EA stimulation (post) as compared to before stimulation. Therefore, the effects of EA stimulation can be confirmed with the increase in ST. In our results, there were four subjects (No. 2, No. 4, No. 6, and No. 13) whose values showed negative values. If the average of the change ratio was not positive, there may be a possibility that the technical level of the partner (who gave EA stimulation to the subjects) was not adequate. Thus, this average ratio could be applied as a new scientific index of technical assessment in acupuncture training/education.

Many studies have shown that acupuncture stimulation encourages release of vasodepressor materials, such as substance $\mathrm{P}$ or calcitonin gene related peptide (CGRP) [6] [7].

Needless to say, EA stimulation increases ST because it repeatedly causes a twitch response on the tibialis anterior during the experiment period. This muscular twitch response could aid "muscle pump", which constantly constricts/dilates blood vessels. It might play an important role in venous circulation. These effects of EA were confirmed using a rat model by Sheriff DD [14]. In this way, Causey L and his colleagues established a theoretical frame work of the effectiveness of EA [15]. In short, we believe that EA elevates ST because it increases local blood volume of blood circulation. Such effects were also confirmed by Lu et al. [16].

Thus, the effects of EA can be shown by monitoring of ST, so that we can bring monitoring of ST into training/education of EA as a new index of technical assessment. Moreover, ratio of changes might be criteria for retraining. These features offer a new possibility in the assessment of acupuncture techniques.

Our study has several limitations. First, we need to determine why four students showed a decrease in ST. Second, there was individual variability (responsiveness or sensitivity). Moreover, we had a limited number of participants; therefore, further research (e.g., larger-scale studies, adjustment of gender differences, BMI, sports, drink and smoking, and other age subjects) is required to support these findings. These would be valuable factors in the assessment of any future study.

\section{Acknowledgements}

The authors thank all of the study participants. We thank for editing a draft of this manuscript Ms. Shirley Horii. 


\section{References}

[1] Dorfer, L., Moser, M., Spindler, K., Bahr, F., Egarter-Vigl, E. and Dohr, G. (1998) 5200-Year-Old Acupuncture in Central Europe? Science, 282, 242-243. https://doi.org/10.1126/science.282.5387.239f

[2] Ma, K. (1992) The Roots and Development of Chinese Acupuncture: From Prehistory to Early 20th Century. Acupuncture in Medicine, 10, 92-99.

https://doi.org/10.1136/aim.10.Suppl.92

[3] Mori, H. and Sasaki, K., Eds. (2009) Shinkyu Kisojissyuu Noto (Fundamental Workbook of Clinical Acupuncture and Moxibustion). Ishiyaku Publishers, Inc., Tokyo, 13-28.

[4] Japan College Association of Oriental Medicine (2016) 1-8. http://www.toyoryoho.or.jp/practical_exam/detail.php?id=1

[5] Jansen, G., Lundeberg, T., Klartansson, J. and Samuelson, U.E. (1989) Acupuncture and Sensory Neuropeptides Increase Cutaneous Blood Flow in Rats. Neuroscience Letters, 97, 305-309. https://doi.org/10.1016/0304-3940(89)90615-0

[6] Kashiba, H. and Ueda, Y. (1990) Acupuncture to the Skin Induces Release of Substance P and Calcitonin Gene-Related Peptide from Peripheral Terminals of Primary Sensory Neurons in the Rat. The American Journal of Chinese Medicine, 19, 189-197. https://doi.org/10.1142/S0192415X91000260

[7] Sato, A., Sato, Y., Shimura, M. and Uchida, S. (2000) Calcitonin Gene-Related Peptide Produces Skeletal Muscle Vasodilation Following Antidromic Stimulation of Unmyelinated Afferents in the Dorsal Root in Rats. Neuroscience Letters, 283, 137-140. https://doi.org/10.1016/S0304-3940(00)00932-0

[8] Felder, D., Russ, E., Montgomery, H. and Horwitz, O. (1954) Relationship in the Toe of Skin Surface Temperature to Mean Blood Flow Measured with a Plethysmograph. Clinical Science (Lond), 13, 251-256.

[9] Hertzman, A.B. (1953) Some Relations between Skintemperature and Blood Flow. American Journal of Physical Medicine, 32, 233-251. https://doi.org/10.1097/00002060-195308000-00005

[10] Kubota, T., Mori, H., Morisawa, T., Hanyu, K., Kuge, H., Watanabe, M. and Tanaka, T.H. (2016) Electric Acupuncture Stimuli (EA) Changes Pupil Diameter (PD), Muscle Blood Volume (MBV) and Skin Blood Flow (SBF) of the Tibialis Anterior. International Conference of World Federation of Acupuncture-Moxibustion Societies Tokyo/Tsukuba, 474.

[11] Mori, H., Kuge, H., Watanabe, M. and Tanaka, T.H. (2016) Does Electroacupuncture Influence Skin Temperature, Skin Blood Flow, and Muscle Blood Volume among Stimulation Sites? Medical Acupuncture, 28, 325-330. https://doi.org/10.1089/acu.2015.1115

[12] Mori, H., Kuge, H., Watanabe, M. and Tanaka. T.H. (2015) Near-Infrared Spectroscopy and Hydrogen Clearance: Is There Any Difference between These Two Methods of Muscle Blood Volume Estimation? Acupuncture in Medicine, 33, 166-167. https://doi.org/10.1136/acupmed-2014-010672

[13] Mori, H., Kuge, H., Tanaka, T.H. and Taniwaki, E. (2014) Influence of Different Durations of Electroacupuncture Stimulation on Skin Blood Flow and Muscle Blood Volume. Acupuncture in Medicine, 32, 167-171. https://doi.org/10.1136/acupmed-2013-010422

[14] Sheriffn, D. (2003) Muscle Pump Function during Locomotion: Mechanical Coupling of Stride Frequency and Muscle Blood Flow. American Journal of Physiology. 
Heart and Circulatory Physiology, 284, H2185-H2191.

https://doi.org/10.1152/ajpheart.01133.2002

[15] Causey, L., Cowin, S.C. and Weinbaum, S. (2012) Quantitative Model for Predicting Lymph Formation and Muscle Compressibility in Skeletal Muscle during Contraction and Stretch. Proceedings of the National Academy of Sciences, 109, 9185-9190. https://doi.org/10.1073/pnas.1206398109

[16] Lu, C., Jan, C.Y.M., Li, T.C. and Hsieh, L. (2009) Electroacupuncture Induces Differential Effects between Yin and Yang: A Study using Cutaneous Blood Flow and Temperature Recordings of the Hand's Dorsum and Palm. The American Journal of Chinese Medicine, 37, 639-645. https://doi.org/10.1142/S0192415X09007120 